Military Technical College Kobry El-Kobba

Cairo, Egypt

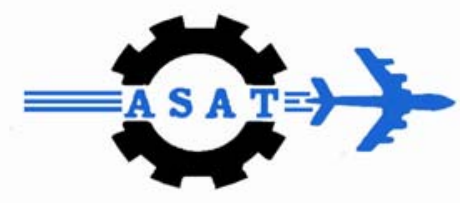

12-th International Conference

on

Aerospace Sciences \&

Aviation Technology

\title{
REAL-TIME IMPLEMENTATION OF DIGITAL RADAR SIGNAL PROCESSOR USING G-LANGUAGE
}

\author{
Sameh G.S*, Mamdouh H.I*, and Fawzy I. A.G**
}

\begin{abstract}
Recently, real-time radar signal processing computation enjoys a significantly high level of development and utilization. It has its own special class of applications that demands extremely high computational performance and very fast real-time response.

In this paper, radar signal processing technique can be implemented in real time using Graphical Language software (G-Language). The radar video signals are acquired and digitized using data acquisition (DAQ) card. These digital signals can be enhanced using one of radar signal processing techniques such as Moving Target Indicator (MTI).

The processed signals are converted into analog signals using the same DAQ card, and displayed on a radar monitoring system. This is the first step to design and implement a digital radar system. In other word, many of radar signal processing techniques such as Moving Target Detector (MTD), Constant False Alarm Rate (CFAR) and Integrator can be implemented digitally using this software algorithm instead of existing hardware digital signal processing circuits.

Using G-language in radar signal processing has many advantages such as simplicity, fast development, low cost, and upgradeable.

Consequently, a huge radar sub-systems used for radar signal processing can be replaced simply by a Personal Computer (PC), DAQ card, and G-Language software. The proposed approach was tested and compared with laboratory radar trainer system.
\end{abstract}

\section{KEYWORDS:}

Radar system design; Radar signal processing; Moving Target Indicator(MTI).

* Egyptian Armed Forces

** Prof. Dr. Fawzy Ibrahim Abdel Ghany, Chairman of communication Department, Misr International University (MIU), Cairo, Egypt. 


\section{INTRODUCTION}

Radar digital signal processor is a basic module in the radar sub-systems. Its performance evaluates the over all radar system performance. Currently, signal processing technologies are based on solutions, namely, Application-Specific Integrated Circuit (ASIC) [1], or programmable processors [2], or Digital Signal Processor (DSP). The signal processing dedicated solutions usually possess a set of software tools and/or application programming interfaces (APIs) to support a variety of common processing tasks [3]. In this paper, one of radar signal processing techniques cam be implemented in real - time using software (G-language).

\section{PROBLEM STATEMENT}

The main objective of this paper is to replace MTI technique by G-language algorithm. This proposed algorithm will be designed and evaluated with a radar trainer system (Labvolt radar).

\section{REAL-TIME IMPLEMENTATION}

The main problem in real-time implementation of any signal processing techniques is the acquiring data without any loss. Acquiring data must be carefully studied before the technique implementation.

\subsection{Acquiring radar signals}

The useful radar signals were extracted from the radar system such as In-phase (IChannel) signal, Quadrature phase (Q-Channel) signal and Trigger (PRF) signal. Amplitude of both I-and Q-channels are nearly bipolar \pm 0.2 Volt. Three values of PRF used 144, 216, and $288 \mathrm{~Hz}$. For this proposed algorithm, $144 \mathrm{~Hz}$ will be used (7ms pulse repetition interval PRI) [4].

To implement a PC-based radar signal processor, NI-PCI6071E DAQ card is used, it has the following main characteristics:

(1) multi-analog input channels up to 64, (2) two analog output channels, (3) input buffer First-In-First-Out (FIFO $=512$ samples) and output buffer (FIFO = 2048 samples), (4) 12-bit resolution of input/output signal (5) maximum analog input sampling rate $(1.25 \mathrm{MHz})$, and (6) Maximum analog output sampling rate $(1 \mathrm{MHz})$. The DAQ configured through application level software, such as NI-DAQ, Component Works, LabVIEW, LabWindows/CVI, and Virtual Bench.

Fig.1 represents the proposed general block diagram of PC based digital signal processing algorithm, describing the radar analog input signals, Analog-to-Digital Converter (ADC), input circular buffer, the proposed digital signal processor, output circular buffer, Digital-to-Analog Converter (DAC) .

The implementation of the signal processor requires synchronization among radar signals parameters, DAQ card configuration parameters, and the programming algorithm using G-language. It is a difficult process that requires careful investigation of these parameters. The configuration of DAQ card and G-language with the radar parameters is done as follow [5]: 


\subsubsection{Analog Input (Al) Interface}

Buffering:

The simple buffering techniques in many DAQ applications can be utilized, but there are some applications where these techniques are not appropriate. If it is desired to acquire data over long periods of time (as in this technique), simple-buffered techniques are not suitable. For these types of applications, circular buffer should be set up to store acquired data in memory. Using circular buffer, data is acquired continuously in the background while Labview software retrieves the acquired data.

If the acquired data is not received from the circular buffer fast enough, some of unread data will be overwritten by newer data. Also, if the acquired data is coming to the buffer faster than it reads by the software, Labview returns an error code "overwrite Error". so this problem can be solved by adjusting one of these parameters: (a) Input buffer size, (b) Scan rate (it determines how many times per second Labview acquiring data from channels), and (c) Number of scans to read at a time ( refers to the number of data acquisitions or readings to acquire from each channel in the channel string).

Type of acquisition:

There are two kinds of acquisition such as: Synchronous and Asynchronous acquisition, also data may be acquired in finite mode or in continuous mode.

The main advantage of acquiring data as continuous and circular buffered is that the manipulation of acquired data can be controlled by Al Read Virtual Instrument (VI). But one limitation is done where the acquisition is synchronous. Once the AI Read VI called, any other tasks cannot be performed until the AI Read VI returns the acquired data. So, asynchronous acquisition is suitable for these applications.

\subsubsection{Analog Output (AO) Interface}

Labview writes an array of output generateg values to the analog output channels at a rate specified by update rate. When the waveform data is too large to fit in a memory buffer or if it is constantly changing, circular buffer can be used to generate the data (as in this technique).

The following steps are used to configure DAQ card with G-language algorithm:

- Three analog signals extracted from the radar trainer system which are the target signals $\mathrm{I}$ and $\mathrm{Q}$ in addition to Trigger signal which used for timing. These signals are input to NI-PCI6071E DAQ card.

- The total number of the samples at a time (patch) from one channel equals the actual buffer size of the DAQ card multiplied by integer factor $(n)$, where $n=2 i$, $i=1,2,3, \ldots \ldots$.

- The practical buffer size which must be multiple of number of total samples taken at a time where:

Minimum buffer size required $=$ Number of total samples 2 .

Maximum buffer size required=available memory in computer RAM, so a computer with extended memory must be used.

- The sampling rate $\left(f_{s}\right)$ controls the number of samples in one PRI to get the total number of samples at a time (patch). So,

Number of patches $=\frac{\text { Total number of samples taken at a time }}{\text { Number of samples in one PRI }}$ 
Sampling frequency $=$ No. of samples in one $T_{r}{ }^{*} P R F\left(f_{r}\right)$.

For every PRF of the radar system, there will be different sampling frequency or sampling rate at constant total number of samples at a time, as shown in Table 1.

- For programming, 3-while loops are used. The first loop is used to acquire the analog input signals I-channel, Q-channel, trigger signal $\left(T_{r}\right)$ and noise from nosie generator. The second loop describes the proposed algorithm designed with Glanguage. The processed signals can be monitored on the graphical user interface of G-language (GUI). The third loop is used to generate the output signal after processing in addition with the trigger signal to ensure the synchronization between the outputs processed video signal and Trigger.

- Queuing function is used as FIFO to transfer digital data of analog input from the master loop or first loop to the second loop. The processed signals from the second loop will be transferred to the third loop without any loss of data.

- Output video radar signal is generated on the first output channel of DAQ card with update rate equals to the sampling frequency of input signals. The output buffer size must equals to double of the total number of samples (patch) to be taken at a time.

- Scan back log output of Al Read.VI is tested which indicates how many data remains in the buffer after collecting the required data.

- The proposed algorithm delay-time is multiple of PRI (No. of total samples taken at a time ${ }^{\star} T_{s}$ ) which is equal to the time require for processing. It is max value of time to acquire data without loss or overwritten.

Fig. 2 represents simple flow chart to specify all data required for acquiring data from analog input channels to be displayed either on GUI or on the radar monitoring system.

\subsection{Moving Target Indicator (MTI) algorithm}

Moving Target Indicator is one of signal processing technique in radar systems. It is a technique that enhances the detection and display of moving radar targets by suppressing fixed targets. MTI consists of single, double or third delay line canceller. The simplest one according to design is the single delay canceller (or two- pulse canceller) [6]. The single delay canceller is implemented by software using Glanguage according its circuits in Fig.3. The delay period is done using software with any other mathematical tools. According to the following equation:

$$
Y(t)=X(t)-X\left(t-T_{r}\right)
$$

In digital domain, the above equation will be:

$$
Y(n)=X(n)-X\left(n-Z^{-1}\right)
$$

\section{4- RESULT ANALYSIS}

After investigating all radar system parameters, DAQ card parameters and the configurations with labview, the following design parameters are adopted:

- Total number of samples taken through one channel $=$ actual buffer size * $n$.

Where $n=2 i, i=1,2,3 \ldots$ choose $n=4$.

$\therefore$ No. of samples to taken at a time (patch) $=512 * 4=2048$ samples. 
- practical buffer size $=$ No. of total samples at a time ${ }^{*} \mathrm{n}$

Where $n=2 i, i=1,2,3 \ldots \ldots$

Minimum buffer size required $=2048 * 2=4096$ samples.

Maximum buffer size required = available memory in computer RAM, so computer with extended memory must be used.

For more safety take $\mathrm{n}=64$.

$\therefore$ Practical buffer size $=2048 * 64=131072$ samples.

- If total number of samples to be taken (patch) $=2048$ samples,

If number of samples in one PRI $\left(T_{r}\right)$ is chosen to be 128 sample,

Then, number of packets (patches) $=\frac{2048}{128}=16$ packets.

Sampling frequency $=$ No. of samples in one $T_{r}{ }^{*} P R F\left(f_{r}\right)$

$\therefore$ Sampling frequency $\left(\mathrm{f}_{\mathrm{s}}\right)=128 * \mathrm{f}_{\mathrm{r}}$.

If PRF $=144 \mathrm{~Hz}$ as in utilized radar system, then sampling frequency $=18432 \mathrm{~Hz}$.

Fig.4 represents a simple figure of the total number of samples at a time (patch) and number of samples at one PRI (Pulse repetition Interval).

- The proposed algorithm (MTI) is designed using this approach software (Glanguage) according to Equ. (2). This algorithm is designed in DSP loop and the evaluation of this algorithm is done in output loop (third loop).

- Update rate, which is the output frequency of signal waveform (its value equal to the sampling frequency of input signal) i.e. update rate $=18432 \mathrm{~Hz}$.

- Buffer size of the output signal waveform must be equal double of the total number of samples (patch) to be taken at a time i.e. (4096 samples) because, if the output buffer size is greater than this value, time required to fill FIFO is large to generate output signal.

- The proposed algorithm time-delay is multiple of PRI (No. of total samples taken

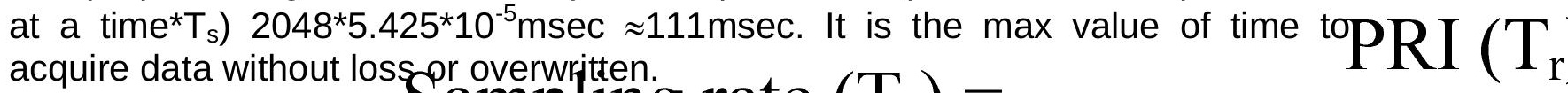

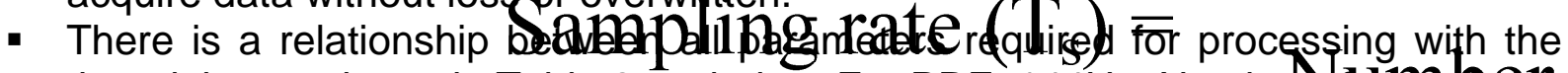

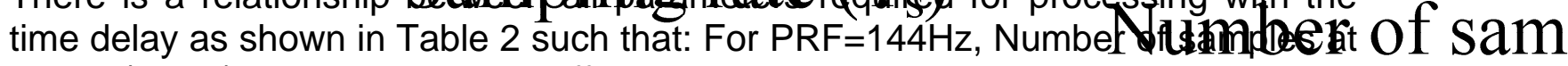
a time $($ patch $)=2048$, practical buffer size used $=131072$ samples.

- Fig.4 represents the whole connections of the proposed algorithm with the applied radar system. Radar monitor display and oscilloscope are used to compare between the analog output video signal from the trainer radar system and analog output video signal from the proposed algorithm.

- Fig.5 represents the relationship between the probability of detection $\left(P_{d}\right)$ versus signal -to-noise ratio (SNR) for the trainer radar system and the proposed algorithm technique at constant probability of false alarm $\left(\mathrm{P}_{\mathrm{fa}}=10^{-4}\right)$.

- Fig.6 represents the probability of detection $\left(P_{d}\right)$ versus signal -to-noise ratio (SNR) for the proposed algorithm at different probabilities of false alarm $\left(10^{-2}, 10^{-4}\right)$

- Fig.7 represents the butterfly of the two videos.

- Fig.8 represents the video signal of the proposed algorithm using PPI.

\section{5- CONCLUSION}

Radar signal processor algorithm such as MTI can be implemented in real-time using DAQ card. The performance of the proposed algorithm is similar to that of the radar 
trainer system for single delay-line canceller. Double delay-line canceller can be implemented with this technique.

This proposed algorithm has been developed using G-Language; its advantages are simplicity, fast development, low cost, and upgradeable.

The proposed approach was tested and compared with laboratory radar trainer system which has nearly the same performance. So, any signal processing module can be replaced be PC and software (G-language). So, huge radar hardware subsystems used for radar signal processing and monitoring systems can be replaced simply by a PC, DAQ card, and G-Language.

This algorithm can be used in any real radar system given its parameters. So, for a real radar system which has pulse width about $1 \mu \mathrm{sec}$ and PRI about $1 \mathrm{msec}$, the sampling frequency will be greater than $2 \mathrm{MHz}$. This can be achieved using Glanguage in addition to DAQ card which has sampling rate up to $20 \mathrm{MHz}$.

So, the radar signal processing techniques MTD, CFAR and integrator can be implemented using this approach. This is the main topics of the current research.

\section{REFERENCES}

[1] P. Pirsch, H.J. Stalberg, VLSI implementation of image and video multimedia processing systems, IEEE Transactions on Circuits and Systems for Video Technology 8 vol.7, pp. 878-891, 1998.

[2] A.Y. Wu, K.J. Ray Liu, A. Raghupathy, "System architecture of an adaptive reconfigurable DSP computing engine", IEEE Transactions on Circuits and Systems for Video Technology 8 , vol.1,pp.54-73,1998.

[3] I.Y. Soona, C.K. Yeob, ${ }^{*}$, H.C. Nga, " An analog video interface for generalpurpose DSP", school of Electrical and Electronic Engineering, Nanyang Technological University, Nanyang Avenue, Singapore, November 2000.

[4] LAB-VOLT LTD., Radar Training Lab volt volume 1,2,3, Technical Publications Department, "Radar Basic System ", First Edition, December Lab-Volt Ltd (1992).

[5] National Instrument, "Labview Data Acquisition Basics Manual", National Instrument Corporate Headquarters, Austin, Taxas, USA, January 2000 Edition.

[6] Mark A. Richards, Ph.D. "Fundamentals of Radar Signal Processing", Georgia Institute Technology, ch.5, 2005. 


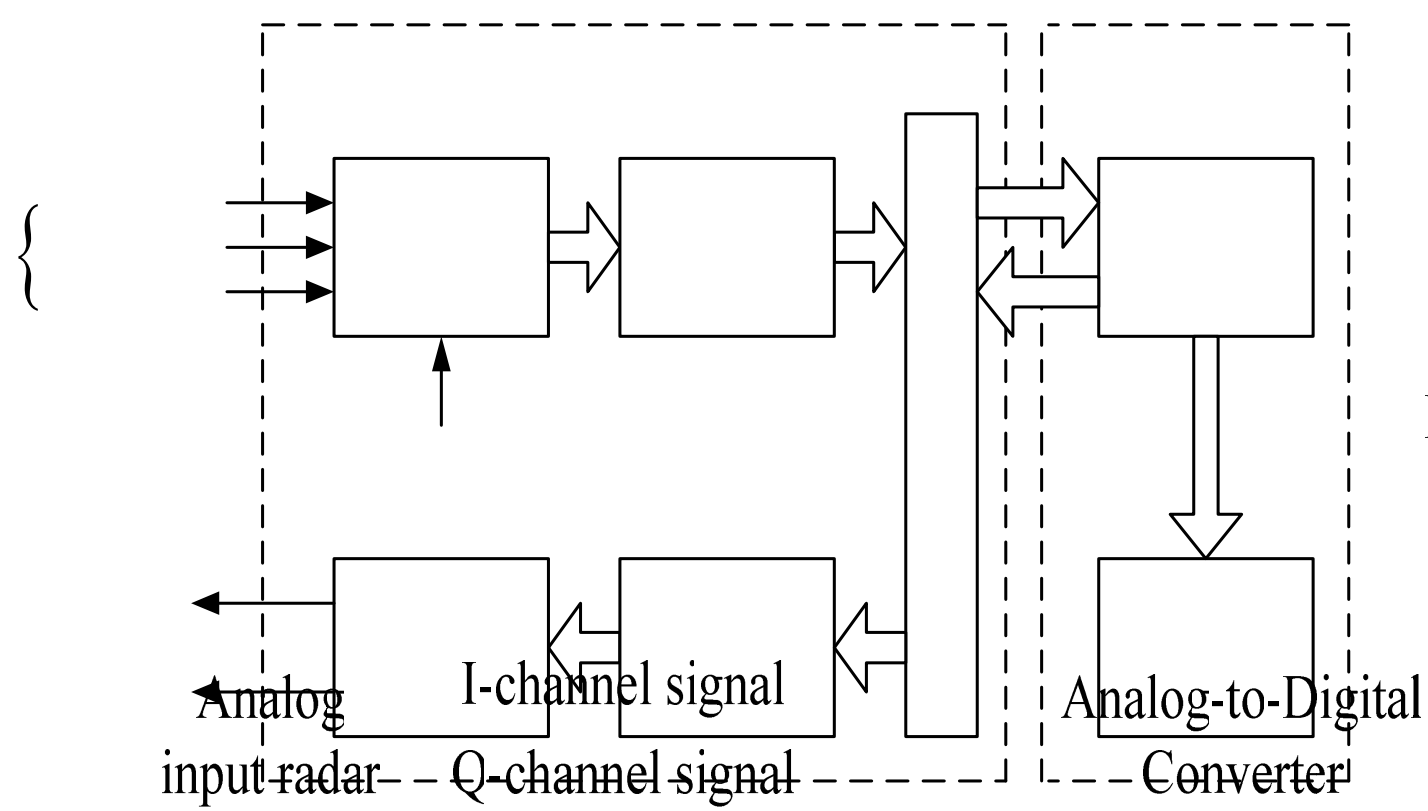

DAQ card cor

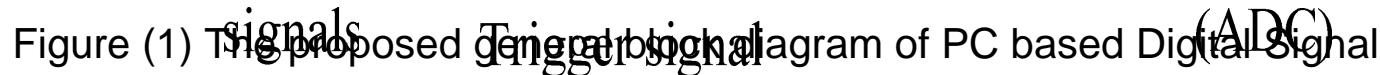
Processing (DSP) algorithm.

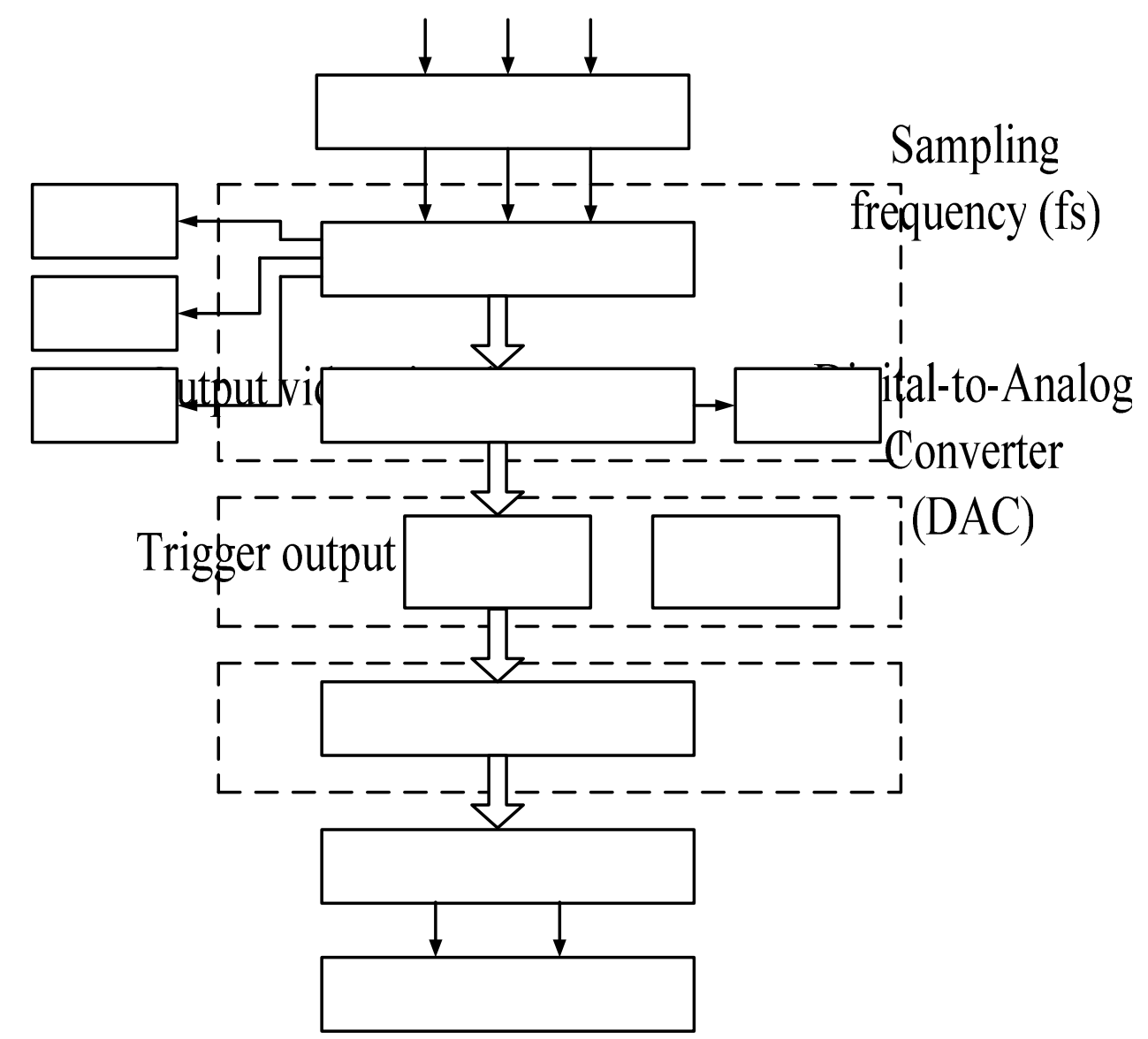

Figure (2) Flow chart of the proposed algorithm with all data required. 


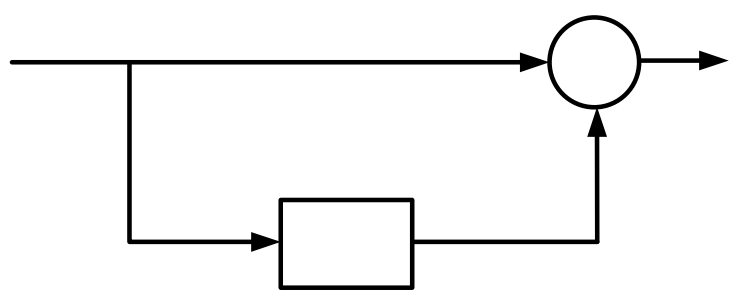

Figure (3) Flowgraph of MTI algorithm (single delay canceller)[7].

$\mathbf{X}(\mathbf{n})$

Table 1 Relations between number of samples in one period of $T_{r}$, number of packets, and sampling frequency

\begin{tabular}{|c|c|c|c|c|}
\hline $\begin{array}{l}\text { Number of } \\
\text { samples in one } \\
\text { PRI }\end{array}$ & $\begin{array}{c}\text { Number of } \\
\text { packets }\end{array}$ & $\begin{array}{c}\mathrm{f}_{\mathrm{s}}(\mathrm{Hz}) \text { at } \\
\mathrm{PRF}=144 \mathrm{~Hz}\end{array}$ & $\begin{array}{c}\mathrm{f}_{\mathrm{s}}(\mathrm{Hz}) \text { at } \\
\mathrm{PRF}=216 \mathrm{~Hz}\end{array}$ & $\begin{array}{c}\mathrm{f}_{\mathrm{s}}(\mathrm{Hz}) \text { at } \\
\mathrm{PRF}=288 \mathrm{~Hz}\end{array}$ \\
\hline 8 & 256 & 1152 & 1728 & 2304 \\
\hline 16 & 128 & 2304 & 3456 & 4608 \\
\hline 32 & 64 & 4608 & 6912 & 9216 \\
\hline 64 & 32 & 9216 & 13824 & 18432 \\
\hline 128 & 16 & 18432 & 27648 & 36864 \\
\hline 256 & 8 & 36864 & 55296 & 73728 \\
\hline
\end{tabular}

Table 2 Relations between numbers of samples in one period of $T_{r}$, sampling frequency, number of packets and delay-time.

\begin{tabular}{|c|c|c|c|}
\hline $\begin{array}{c}\text { Number of samples } \\
\text { in one PRI }\end{array}$ & $\begin{array}{c}\text { Number of } \\
\text { packets }\end{array}$ & $\begin{array}{c}\mathrm{f}_{\mathrm{s}}(\mathrm{Hz}) \text { at } \\
\mathrm{PRF}=144 \mathrm{~Hz}\end{array}$ & $\begin{array}{c}\text { Time-delay of } \\
\text { first loop } \\
(\mathrm{msec})\end{array}$ \\
\hline 8 & 256 & 1152 & 1792 \\
\hline 16 & 128 & 2304 & 896 \\
\hline 32 & 64 & 4608 & 448 \\
\hline 64 & 32 & 9216 & 224 \\
\hline 128 & 16 & 18432 & 112 \\
\hline 256 & 8 & 36864 & 56 \\
\hline
\end{tabular}

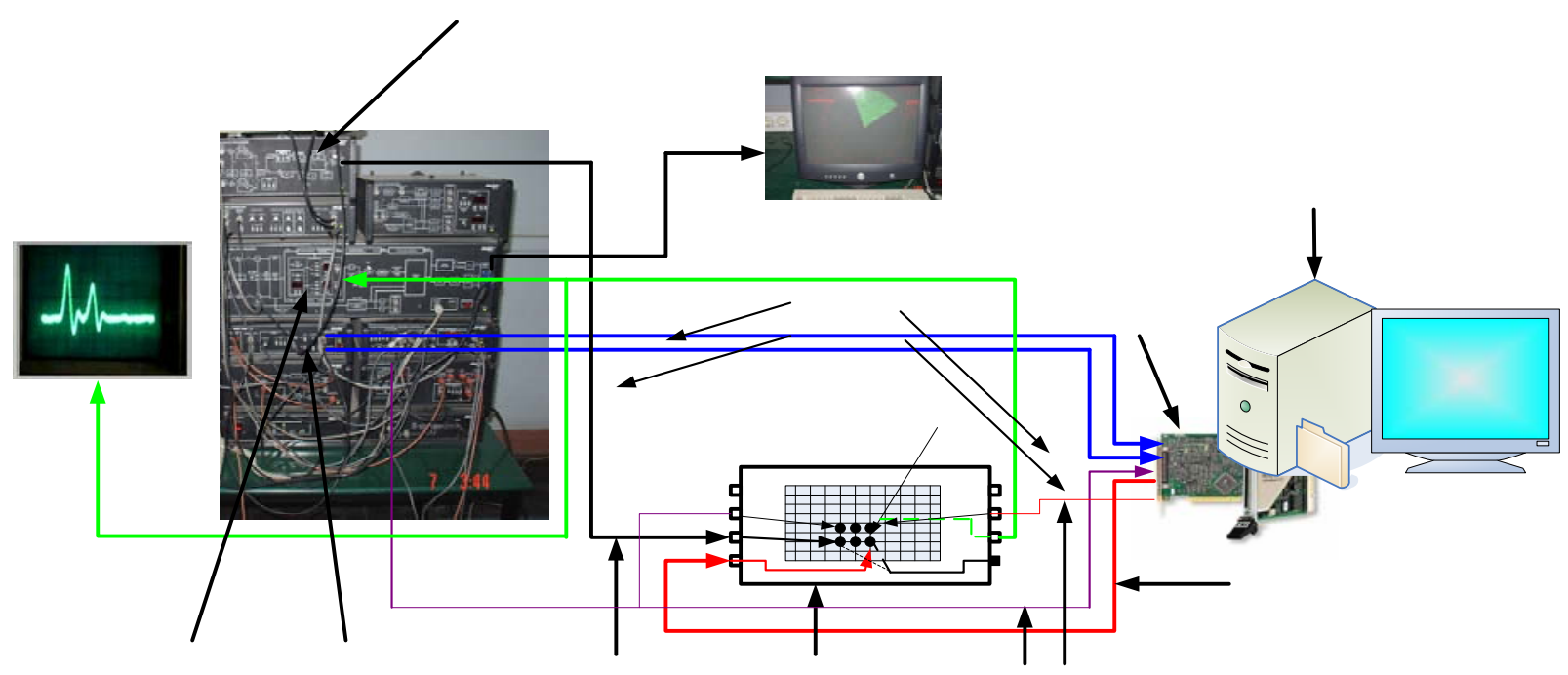

Figure (4) Connections of NI-PCI 6071E with radar system. 


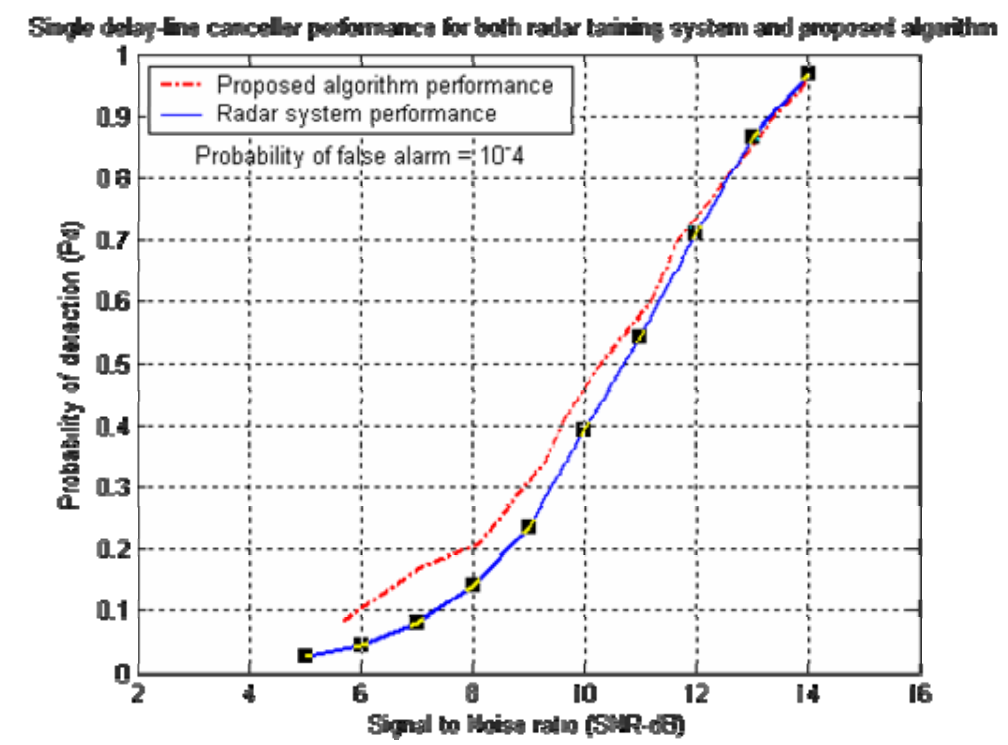

Figure (5) Probability of detection (Pd) versus Signal-to-Noise Ratio (SNR) at constant probability of false alarm $\left(10^{\wedge}-4\right)$.

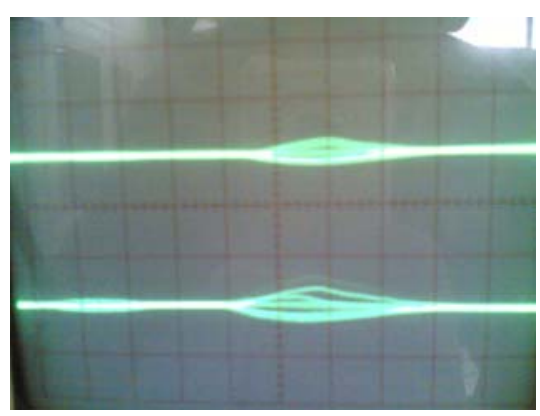

Figure (7) Video output due to butterfly on oscilloscope screen from: 0 Output video from the radar system on the top figure.

o Output video from the proposed algorithm on the bottom figure.

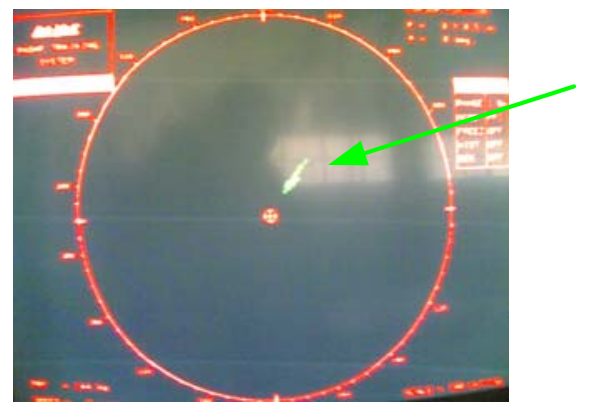

Figure (9) Video output from proposed algorithm for moving target 